\title{
Novel CADe/CADx System for Lung Nodules Segmentation and Classification on Computed Tomography Images
}

\author{
Vijayalaxmi Mekali, Girijamma H. A
}

\begin{abstract}
Detection and classification of different types lung nodules poses major challenges in medical diagnosis routine. Classification of segmented nodules based on extracted hybrid features of segmented nodules have shown remarkable performance. Recently deep features alone and also with combination of hybrid features have improved nodules classification. In this research work new CADe/CADx system is proposed for detection and classification of Well Circumscribed Nodules, Juxta Vascular Nodules and Juxta Pleural Nodules. In nodules detection part, algorithms proposed in our previous work were used. Classifiers decision fusion based new nodules classification system is proposed. Four set of hybrid features and deep features using Convolution Neural Network are considered from segmented nodules. Hybrid features set consist of twenty four shape features, six GLCM features in four direction with a distance of two, six First Order Statistic features and twelve energy features. Five individually trained Probabilistic Neural Networks by all five set features separately used in nodule classification. In classification process all five classifiers decisions are fused at 2-level, 3-level, 4-level and 5-level. The proposed system achieved highest performance with 5-level fusion compared with other level fusions. System was evaluated on CT images of LIDC database with consideration of 2669 lung nodules of malignancy rate 1 to 5 . Based on malignancy rate 2669 nodules are grouped as dataset 1 and dataset 2 with nodules of malignancy rate 1, 2, 3 and 3, 4,5 respectively. The 5-level decision fusion achieved highest accuracy of 95.72, sensitivity of 95.52, specificity of 95.79 and Area Under Curve of 96.21 for dataset 1 and accuracy of 92.54, sensitivity of 90.48, specificity of 94.63 and Area Under Curve of 92.69 for dataset 2.
\end{abstract}

Keywords: Computed Tomography, Computer Aided Detection/Diagnosis, Convolution Neural Network, Lung cancer and Lung Nodule Classification.

\section{INTRODUCTION}

All over the globe mortality rate of lung cancer is very high as compared with other types of cancers such as prostate, brain, breast, cervical cancer. According to World Lung Cancer Day 2019 facts and figure, lung cancer new cases have risen to 2.09 million and 1.76 million deaths all over the world. Early stage detection and classification of lung cancer is acceptable solution to reduce the mortality rate. Lung cancer is heterogeneous disease as it appears at different

Revised Manuscript Received on December 12, 2019.

* Correspondence Author

Vijayalaxmi Mekali*, Department of Computer Science and Engineering, Kammavari Sangham Institute of Technology, Visvesvaraya Technological University, Bangalore, India. Email: duruth.viju@gmail.com

Dr. Girijamma H. A, Department of Computer Science and Engineering, R. N. S Institute of Technology, Visvesvaraya Technological University, Bangalore, India.. Email: girijakasal@gmail.com locations in lungs attached with different types of external structures with different calcification rate. Lung cancer can be classified into different type according its site of origin, cells size, and attachment of external structures, malignancy rate and solidity. Well Circumscribed Nodule (WCN) is round or oval shaped lung nodules appears in center of lung area without any additional tissues attachment. Juxta Vascular Nodule (JVN) is attached with blood vessels and Juxta Pleural Nodule (JPN) attached with lung pleural as shown in Fig. 1. At next level WCNs, JVNs and JPNs are classified into solid, part-solid and non-solid nodules. Based on severity lung nodules are either benign or malignant. Small nodules without cancerous cells are known as benign nodules. Moderate or large size nodules with cancerous cells are known as malignant (have potential to spread to other sites). Malignancy of malignant lung nodules indicates potential of these nodules to extent to lymph nodes, or to another lung lobe or to other organs like breast, brain, prostate etc. Farthest speared of malignant nodules indicates higher malignancy. Table I shows the characteristics of benign and malignant nodules.

A. Low Dose Computed Tomography (LDCT) and Computer Aided Detection/Diagnosis (CADe/x) system for lung cancer

Medical modalities like X-ray, Computed Tomography (CT), Magnetic Resonance Imaging (MRI), Positron Emission Tomography (PET) and Diffusion Weighted-MRI (DW-MRI) have been playing major role in detection of lung cancer. Among all the medical modalities Low Dose CT (LDCT) is GOLD STANDARD for detection of lung cancer. LDCT generates huge number of images (slices) in one single scans. It is tedious task for radiologist to interpret all the slices to draw useful information about existing nodules. To reduce the burden of radiologist and for accurate detection of lung cancer nodules, medical routines uses Computer Aided Detection/Diagnosis (CADe/x) system to detect and draw precise information about nodules. Benign or malignant nodules classification is done by CADx system and thus it helps the radiologist in treatment plan.

B. Role of Image processing techniques and classifier in lung cancer diagnosis

Image processing techniques such as different types of thresholding methods, Region Growing (RG) method, clustering algorithms, morphological operations, edge 
detection algorithms, active contour models and others have been used in many CAD system by different researchers to detect lung cancer. Classifiers such as ADA boost, Support Vector Machine (SVM), decision tree, and other have been playing a major role in lung cancer CADx system to classify detected nodule into benign and malignant. Currently Artificial Neural Networks (ANN), deep learning techniques such as Convolution Neural Network (CNN), Recurrent Neural Network (RNNs), Long Short-Term Memory Networks (LSTMs), Stacked Auto-Encoders, Deep Boltzmann Machine (DBM) and Deep Belief Networks (DBN) have been a part of CADx system for lung nodules classification.

Table- I: Characteristic of lung nodules

\begin{tabular}{|c|c|c|}
\hline Characteristic & Rating & Remark \\
\hline 1. Calcification & $\begin{array}{l}\text { 2. Popcorn, } \\
\text { 2.Laminated } \\
\text { Solid } \\
\text { 3. Non-central } \\
\text { 4. Central } \\
\text { 5. Absent }\end{array}$ & $\begin{array}{l}\text { Pattern of calcium } \\
\text { deposit in nodule }\end{array}$ \\
\hline $\begin{array}{l}\text { 2. Internal } \\
\text { structure }\end{array}$ & $\begin{array}{l}\text { 1. Soft tissue } \\
\text { 2. Watery 3.Fat,4. } \\
\text { Air }\end{array}$ & $\begin{array}{l}\text { Presence of possible } \\
\text { component in nodule }\end{array}$ \\
\hline 3. Lobulation & $\begin{array}{l}\text { 1. Marked, } 5 . \\
\text { None }\end{array}$ & \\
\hline $\begin{array}{l}\text { 4. Malignancy } \\
\text { rate }\end{array}$ & $\begin{array}{l}\text { 1. Highly unlikely } \\
\text { 2. Moderately } \\
\text { unlikely } \\
\text { 3. Intermediate } \\
\text { 4. Moderately } \\
\text { suspicious } \\
\text { 5. Highly } \\
\text { suspicious }\end{array}$ & $\begin{array}{l}\text { Larger the size of } \\
\text { malignant nodules higher } \\
\text { the Malignancy rate. }\end{array}$ \\
\hline 5. Margin & $\begin{array}{l}\text { 1. Poorly defined } \\
\text { 5. Clearly defined }\end{array}$ & $\begin{array}{l}\text { Describes where the lung } \\
\text { nodules is clearly defined }\end{array}$ \\
\hline 6. Sphericity & $\begin{array}{l}\text { 1. Linear } \\
\text { 3. Ovoid } \\
\text { 5. Round }\end{array}$ & $\begin{array}{l}\text { Describes shape of } \\
\text { nodules }\end{array}$ \\
\hline 7. Spiculation & $\begin{array}{l}\text { 1. Marked, } 5 . \\
\text { None }\end{array}$ & $\begin{array}{l}\text { Spikes along the } \\
\text { boundary of lung nodules } \\
\text { indicates their } \\
\text { malignancy }\end{array}$ \\
\hline 8. Subtlety & $\begin{array}{l}\text { 1. Very subtle } \\
\text { 2. Soberly Subtle } \\
\text { 3. Justly Subtle } \\
\text { 4. Moderately } \\
\text { Obvious } \\
\text { 5. Obvious }\end{array}$ & $\begin{array}{l}\text { Contrast between the } \\
\text { lung nodules and } \\
\text { surrounding nodules. }\end{array}$ \\
\hline 9. Texture & $\begin{array}{l}\text { 1. Solid, 3. Part } \\
\text { solid } \\
\text { 5. Non-solid }\end{array}$ & $\begin{array}{l}\text { Nodules density, solid } \\
\text { nodules -highly with clear } \\
\text { boundary part solid and } \\
\text { non-solid -poorly dense, } \\
\text { difficult to identify } \\
\text { boundary }\end{array}$ \\
\hline
\end{tabular}

\section{RELATED WORK}

As lung cancer detection and classification plays major role in reducing mortality rate of lung cancer, extensive research have been done in implementation of lung cancer CAD systems. But it is still remaining as challenging research area for early detection and classification of lung nodules. Many researchers considered texture features of segmented nodules for classification of nodules as benign or malignant $[12,13]$. Shape features were considered for lung nodules classification in [14, 15]. Morphological operations based nodules classification was proposed in [16, 17]. Gray Level Covariance Matrix (GLCM) based texture features of segmented lung nodules were used in $[18,19]$ for nodules classification. CNN was used in classification process of lung nodules in [20, 21, 22]. In [1] classification of segmented nodules based on textures features of nodules using Gabor filter and Multi Resolution Local Binary Pattern (MRLBP) fused with shape features from singed distance was proposed. Support Vector Machine (SVM) and K-Nearest Neighbor (KNN) classifiers were used to classify segmented nodules as benign or malignant. Performance of proposed method was evaluated on CT images from LIDC database. Author in [2] developed semi-automated CAD system for segmentation of lung nodules from CT images. Features such as shape, margin and textures of segmented nodules were used by SVM classifier to classify nodules. Implemented system was evaluated on 891 images from LIDC. Proposed lung nodules classification model in [35] was based on 66 3D features from segmented nodules. Three classifiers SVM, Navie Bayes and Linear Discriminant Analysis (LDA) were used for classification. In this experiment Area Under Curve (AUC) of $0.94,0.90,0.99$ was achieved by three classifiers respectively. In [3] lung nodules classification system was designed based on Artificial Neural Network (ANN). In [4] authors provided clear discussion on currently available different lung nodules detection and classification techniques. Authors in [5] developed lung nodules classification model using fused shape, texture and deep learned features of segmented nodules. First set was texture features calculated from GLCM, second set was Fourier shape features and third set was Deep CNN (DCNN) features. Three Ada Boosted Back Propagation Neural Network (BPNN) were trained separately with three features set. Finally output of all three BPNNs are fused for final classification of nodules. Proposed method was evaluated on LIDC images and AUC of 96.65\%, 94.45 and $81.24 \%$ achieved respectively. New model was

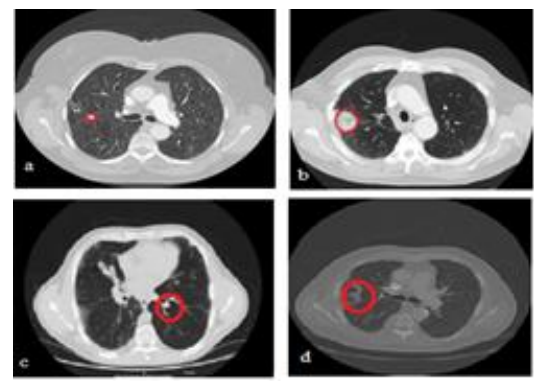

Fig. 1. Different types of lung nodules (a) WCN (b) JPN (c) JVN (d) GGO nodule in CT images.

designed in [6] to classify lung nodules as benign or malignant based on Two- step Content Based Image Retrieval (CBIR). In first step $\mathrm{K}$ most similar ROI were extracted using semantic relevance and in second step visual similarity was used to extract the weight of each ROI in first step. Obtained probability was used to classify input nodule as benign or malignant. In [7] new model Deep lung diagnosis model was presented for lung nodules classification. In Deep lung model, 3D faster R-CNN was used for nodule detection, features
Published By:

\& Sciences Publication 
were learned with encoder and detector structure and Gradient Boosting Machine (GBM) with 3D Dual Path Network (DPN) was used for classification. Model was evaluated on CT images from LIDC. Authors in [8] implemented model based on features extracted from DNN fused with raw features extracted from input image. Model was evaluated on 20 subject with 3598 nodules such that 178 malignant nodules and 3420 benign nodules. Model succeeded in achieving accuracy of $95.5 \%, 94.4 \%$, sensitivity of 94.4 and AUC of 0.987. In [9] lung nodules classification system was modeled using fractal features from Fractional Brownian motion model. SVM classifier was used to classify the nodules. $107 \mathrm{CT}$ images of 107 different patients were considered. Model achieved accuracy of $88.82 \%$, sensitivity of 93.92 , specificity of $82.90 \%$ and AUC of 0.9019 . Author designed Neural Network Ensemble (NNE) model in [11] for lung nodules classification. In this model NNE was implemented using multilayer NN with BPNN, Radial Basis Probabilistic Neural Network (RBPNN) and Learning Vector Quantization Neural Network (LVQNN). Output of NNE was considered for Bayesian classifier to classify the nodules. Designed method achieved a classification accuracy of $78.7 \%$. In [10] new lung nodule classification model was developed. Texture features of nodules in CT images were extracted with Shannon and simpson diversity indices. SVM classifier was used to classify the nodules. Accuracy of $92.7 \%$, sensitivity of 85.64 and specificity of 97.89 was achieved.

\section{METHODOLOGY}

Proposed integrated lung cancer CADe/CADx system consist of three stages. Stage-I performs the CT input image pre-processing, segmentation of lung parenchyma and separation of connected lung lobes. Segmentation of lung nodules is achieved with Stage-II. Stage-I and Stage-II together makes CADe system. In Stage-III GLCM based texture features, shape features, First Order Statistics (FOS) features, energy features and deepCNN features of segmented nodules are extracted. Extracted five set of features are used to train five Probabilistic Neural Networks (PNN) individually for classification of nodules. Classification decisions of all five PNNs are fused finally to classify nodules into benign or malignant. Fig. 2 shows the proposed CADe/CADx system for lung nodules segmentation and classification.

\section{A. Segmentation of lung nodules from CT image}

Main objective of lung nodules segmentation procedure is accurate identification of all types of true lung nodules. And thus to provide information about lung nodules to radiologist. Using this valuable information radiologist can plan radiation treatment with an aim of without effecting nearby anatomical structures (lymph nodes) or attached components (attached blood vessels in JVN and pleura in JPN).

Lung nodules segmentation procedure is not same for all types of lung nodules. WCN appears in area of lung without any external attachments. Thus WCN segmentation does not poses much difficulties. But JVN and JPN with attached external components (blood vessel in case of JVN and Pleura in case of JPN) needs typical segmentation procedures. In case of JVN, segmentation must consider removal of attached blood vessel from the segmented nodules. Traditional segmentation procedure for JPN segmentation results in concavity in lung parenchyma, this is due to gray level similarity of attached pleura and nodules and causes exclusion of JPN.

In this work WCNs, JVNs and JPNs Segmentation was achieved with algorithms proposed in our previous work [25], [26], unpublished [27] respectively.

\section{B. Hybrid Feature Extraction}

Effectiveness of CADx system lies in the accurate discrimination of pulmonary nodules into benign or malignant. In our CADx part, fused decision from PNNs was used for accurate classification of segmented nodules as benign or malignant. Four set of hybrid features and 32 deepCNN features were extracted from segmented nodules. Five set of extracted features were separately used to train the five PNNs. Finally decisions of five PNNs were fused to classify nodules. Considered hybrid features set were: Set-S with extracted $232 \mathrm{D}$ and one 3D shape features to determine geometric aspects of nodule, Set-F with six FOS features based on histogram of segmented nodules to analyze intensity characteristics of nodule, Set-G with 24 Gray level features of segmented nodule (six features in direction $0^{\circ}, 45^{\circ}, 90^{\circ}$ and $135^{\circ}$ with distance of 2) that determines texture characteristics of nodule. Set-E with wavelet transformation based twelve Energy Features. Set-D with deep features of segmented nodules extracted from CNN. Shape features of nodules considered in this work are height, width and depth of bounding box containing nodule (S1), area(S2), standard deviation (SD) of area (S3), Perimeter (S4), SD of perimeter (S5), Mean Equivalent Diameter (S6), SD of Equivalent Diameter (S7), Mean Eccentricity (S8), SD of Eccentricity (S9), Extend (S10), SD of Extend (S11), Compactness ( S12), SD of Compactness (S13), Radial Distance (S14), SD of Radial (S15), Roughness (S16), SD OF Roughness (S17), Elongation (S18), SD of Elongation (S19), Convexity (S20), SD of Convexity (S21), Circularity (S22), SD of Circularity (S23) and one 3D feature Sphericity (S24). First order statistic features considered are mean $(\mathrm{F} 1)$, variance $(\mathrm{F} 2)$, Skewness (F3), Kurtosis (F4), Standard Deviation (F5), Entropy (F6). Considered GLCM features are Entropy (G1), Difference of Entropy (G2), Energy (G3), Contrast (G4), Homogeneity (G5) and Correlation (G6). Features in 4 sets are defined as follows in equations 1 to 25 .

$$
\begin{aligned}
& S 4=\sum_{i=1}^{n} d i \\
& \mathrm{~S} 6=2 \sqrt{\frac{\text { Acircle }}{\pi}} \\
& \mathrm{S} 8=\frac{\text { length }(\text { maj oraxis })}{\text { Length(min oraxis })}
\end{aligned}
$$




$$
\mathrm{S} 10=\frac{\text { ROIArea }}{\text { BoundingrectangleArea }}
$$$$
\mathrm{S} 12=\frac{(\text { Perimeter })^{2}}{4 \pi \bullet \text { area }}
$$$$
\mathrm{S} 16=\frac{\text { PerimterofROI }}{\text { Convexhullperimeter }}
$$$$
\mathrm{S} 18=\frac{\text { Widthbox }}{\text { Lenghtbox }}
$$$$
\mathrm{S} 20=\frac{\text { ConvPermeter }}{\text { PerimeterofROI }}
$$$$
\mathrm{S} 22=\frac{4 \pi \bullet \text { area }}{(\text { convPermeter })^{2}}
$$$$
\mathrm{S} 24=\frac{\text { Rincribing }}{\text { Rcircumscribing }}
$$$$
\mathrm{E} 1=\frac{1}{\mathrm{n}} \sum_{\mathrm{i}=1}^{\mathrm{n}} \mathrm{xi}
$$$$
\mathrm{E} 2=\frac{1}{\mathrm{n}-1} \sum_{\mathrm{i}=1}^{\mathrm{n}}(\mathrm{xi}-\mathrm{m})^{2}
$$$$
\text { E8 }=\frac{\sum_{i=1}^{n} \text { fixpi }}{\sum_{i=1}^{n} p i}
$$$$
\mathrm{F} 1=\sum_{\mathrm{i}=0}^{\mathrm{G}-1} \mathrm{IP}(\mathrm{I})
$$$$
\mathrm{F} 2=\sum_{i=0}^{\mathrm{G}-1}(\mathrm{i}-\mathrm{m} 1)^{2} \mathrm{P}[\mathrm{I}]
$$$$
\mathrm{F} 3=\sum_{\mathrm{i}=0}^{\mathrm{G}-1}(1-\mathrm{m} 1)^{3} \mathrm{P}[\mathrm{I}]
$$$$
\mathrm{F} 4=\sum_{\mathrm{i}=0}^{\mathrm{G}-1}(1-\mathrm{m} 1)^{4} \mathrm{P}[\mathrm{I}]
$$

$F 5=\sqrt{\frac{\sum_{i=0}^{M-1} \sum_{j=0}^{N-1}(A[i, j]-F 3)^{3}}{M * N-1}}$

$\mathrm{F} 6=\sum_{\mathrm{i}=0}^{\mathrm{G}-1} \mathrm{P}[\mathrm{I}] \log 2 \mathrm{P}[\mathrm{I}]$

$$
\mathrm{G} 1=\sum_{\mathrm{i}, \mathrm{j}=0}^{\mathrm{G}-1} \mathrm{Pij}(\log \mathrm{Pij})
$$

$$
\begin{aligned}
& \mathrm{G} 2=\sum_{\mathrm{i}=0}^{\mathrm{N}-1} \mathrm{Px}-\mathrm{y}(\mathrm{i}) \log \{\mathrm{Px}-\mathrm{y}(\mathrm{i})\} \\
& \mathrm{G} 3=\sum_{\mathrm{i}} \sum_{\mathrm{j}}(\mathrm{i}-\mathrm{j})^{2} \mathrm{Pij} \\
& \mathrm{G} 4=\sum_{\mathrm{i}, \mathrm{j}=0}^{\mathrm{G}-1}(\mathrm{Pij})^{2} \\
& \mathrm{G} 5=\sum_{\mathrm{i}, \mathrm{j}=0}^{\mathrm{G}-1} \frac{\mathrm{Pij}}{1+(\mathrm{i}-\mathrm{j})^{2}} \\
& G 6=\frac{\sum_{i=1}^{G}\left\{\sum_{j=1}^{G}\{i \cdot j \cdot P i j\}\right\}-\mu x . \mu y}{\sigma x \cdot \sigma y}
\end{aligned}
$$

\section{C. deepCNN Feature Extraction}

For deep features extraction from segmented nodule, LeNet-5 based CNN with three convolution layers (ConL), three pooling layers and two fully connected layers was used. Segmented nodule region of size $32 \times 32$ from the segmented nodule image was considered as an input for deepCNN to learn the segmented nodule features. In this model Rectified Linear Units (RLU) were used in convolution layers and in last two layers. In first and second convolution layers, $7 \times 7$ sized 32 kernels were applied to generate thirty two $2 \mathrm{D}$ features maps of size $32 \times 32$ and sixteen $2 \mathrm{D}$ features maps of size $16 \times 16$ respectively. And 64 kernels of same size were used in third layer to generate sixty four feature maps of size $16 \times 16$. In average pooling layer $(\operatorname{AvgL} 5 \times 5$ kernels were used so that input features maps from convolution layer were get reduces to one and half of original size. Total of 32 deep features from seventh layer were considered. Fig. 3 shows the architecture of CNN used in proposed system. PNN model used in proposed is shown in Fig. 4.

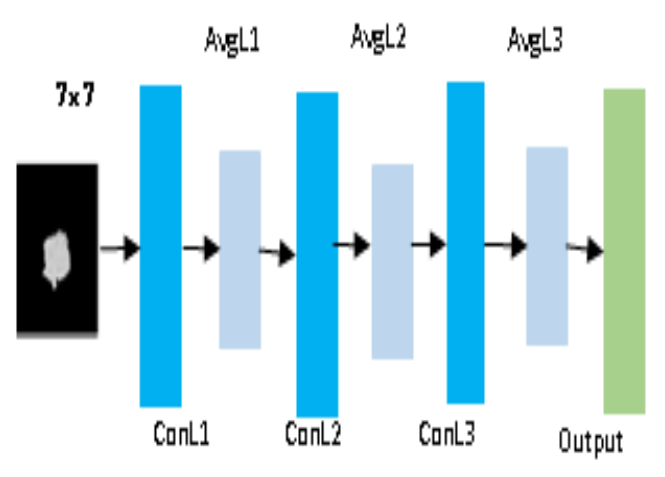

32. 32. 16 $\times 16 \quad 16 \times 16$

Fig.3.CNN model in proposed system 


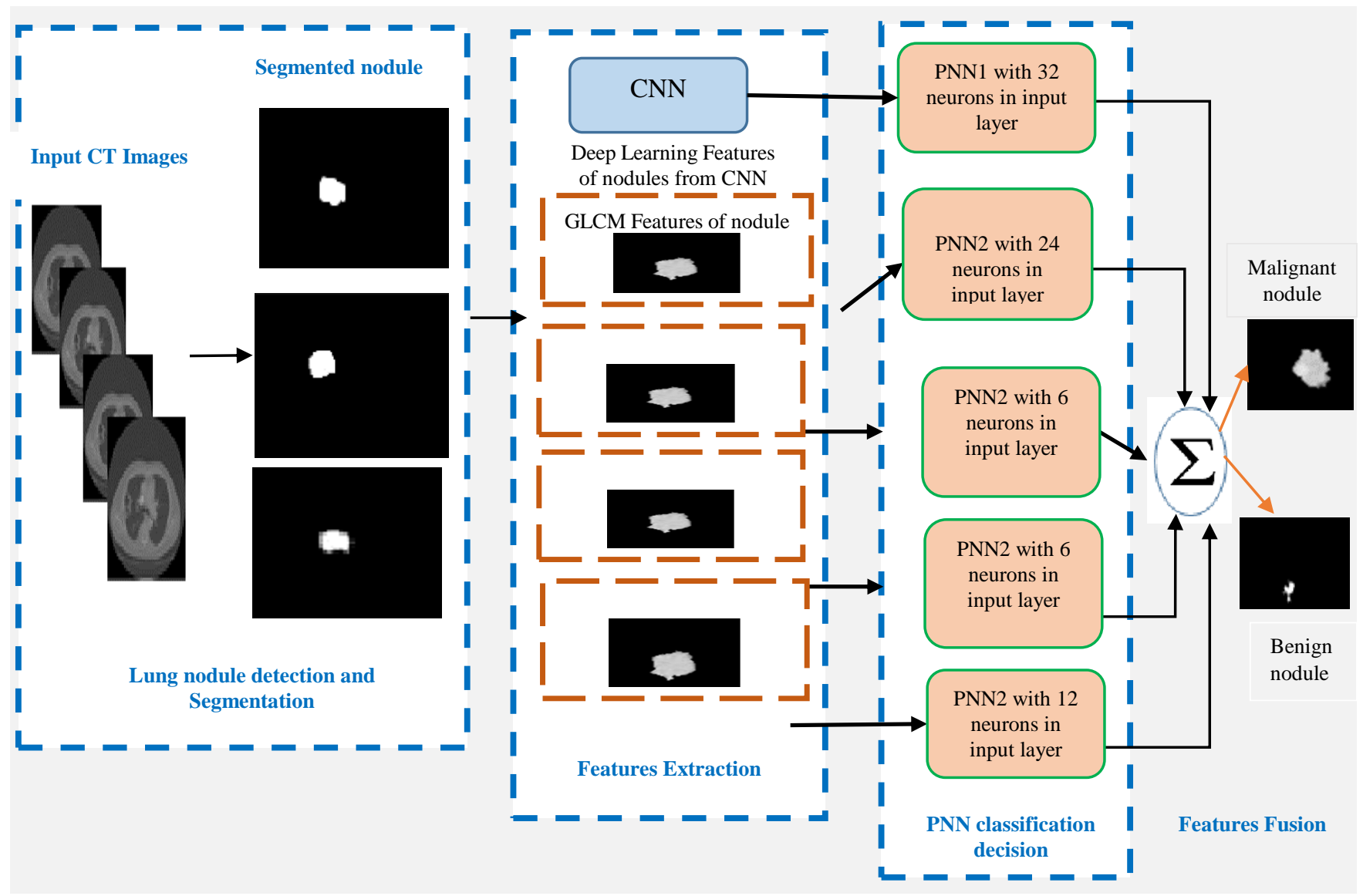

Fig. 2. Architecture of proposed system for classification of lung nodules as benign or malignant

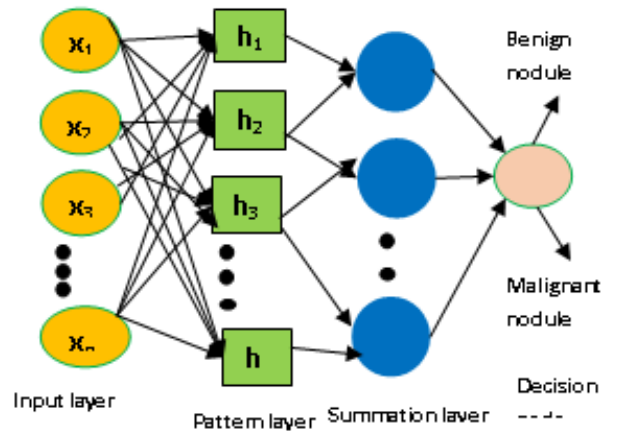

Fig.4.Architecture of Probabilistic Neural Network in proposed system

\section{A. Nodule classification}

In our classification process of CADx system, PNNs fused decision was used for automatic classification of segmented WCNs, JVNs and JPNs as benign or malignant. PNN is feed forward neural network. As it is efficient classifier, it have been used in many classification problem and also in disease classification. Five PNNs were trained separately using four set of hybrid features and deepCNN features. In all five PNNs input layer, pattern layer, summation layer and output layer were used. But different number of neurons in input layer of PNNs were considered. Input layer with 24 neurons in PNN for shape features, 6 neurons in input layer of PNN for FOS

PNN with GLCM features 24 neurons in input layer, and 32 in input layer of PNN with deep features.

\section{B. Description of Data set}

LIDC is international public database of thoracic CT scan images. It was founded by National Cancer Institute (NCI) in 2004, in collaboration with seven academic institutes and eight medical imaging companies. The main aim of the LIDC is to promote the lung cancer detection and diagnosis research globally by providing free web accessible CT scan images of patients suffering from lung cancer. Currently LIDC is maintaining 1018 patients CT scan images. LIDC repository also contains XML file of each scan that provides nodule's spatial locations, annotation, malignancy rate and type. XML file contains details of Nodules $<3 \mathrm{~mm}$ in diameter (not clearly benign), Nodules $>3 \mathrm{~mm}$ and deposits which look like nodules (non-nodules) $>3 \mathrm{~mm}$. For types of nodules $>3 \mathrm{~mm}$ file also contains boundary coordinates. Nodules annotation by up to four radiologist is provided in XML sheet separately $[23,24]$. Nine characteristics of nodules considered with respect to LIDC images along with rating are listed in Table I.

Malignancy rate (MR) given to nodule are 1, 2, 3, 4, 5. In that 1 and 2 rating indicates nodules are benign, whereas 4 and 5 indicates malignant nodules. MR- 3 indicates ambiguity of either being of benign or malignant or can be discarded. In our study total number of 2669 nodules were considered, such that 389 nodules with MR-1, 935 nodules with MR-2, 697 nodules with MR-3, 464 with MR-4 and 184 nodules with MR-5. With this dataset 1 was MR-3 in benign group, with total number of benign nodules 2021 and dataset 2 was MR-3 in malignant group with total 
number of malignant nodules were 1345 .

\section{RESULT AND DISCUSSION}

Proposed system was evaluated CT images from LIDC database. Segmentation of WCNs, JVNs and JPNs was achieved with our previously proposed algorithms [25], [26], unpublished [27]. Segmented results were compared with traditional segmentation method $\mathrm{RG}$ and watershed algorithms. Our methods had achieved highest accuracy in nodules segmentation. In classification process, total number of 2669 nodules were considered. Five PNNs decisions were fused at different level to classify nodules as benign or malignant. Decision of two PNNs were fused in 2-level fusing with different combination of two PNNs decision. Similarly three PNNs and four PNNs decisions were fused in different combinations at 3-level fusing and 4-level fusing. And final fusing, 5-level was fusion of decisions of all five PNNs. Accuracy (Acc), sensitivity (Sen) and specificity (Spe) were computed based on confusion metric as shown in Table II using the equations (26), (27), (28).

Table III and Table IV shows the performance of PNNs in classification of WCNs, JVNs and JPNs at different decision fusing level with dataset 1 and dataset 2. As performance parameters in Table IV indicates that PNNs performance on dataset1 is considerably good at fusion 5-level compared to other level fusions. But PNNs performance at all fusion levels is lesser on dataset 2 compared with its performance on dataset 1. To extend the nodules classification evaluation process, decision fusions results were compared with features fusions at different combination. Computed values for features fusions at different level and comparison with decision fusions at different level are shown in Table VI. Comparison shows that PNNs decisions fusion at 5-level fusion is considerably high in nodules classification as benign or malignant. In Fig. 4 (a) shows CT images and (b) is the lung parenchyma extracted images. Fig. 5 (a) shows the CT images and (b) shows segmented nodules images by proposed algorithm of our previous work.

Table-II: Confusion matrix for proposed system

\begin{tabular}{|c|c|c|}
\hline Confusion metric & $\begin{array}{c}\text { Benign lung } \\
\text { nodules }\end{array}$ & $\begin{array}{c}\text { Benign lung } \\
\text { nodules }\end{array}$ \\
\hline Benign lung nodules & TN & FP \\
\hline $\begin{array}{c}\text { Malignant lung } \\
\text { nodules }\end{array}$ & FN & TP \\
\hline
\end{tabular}

$$
\text { Sensitivity }=\frac{T P}{P}
$$

$$
\text { Specificity }=\frac{\mathrm{TN}}{\mathrm{N}}
$$

$$
\text { Accuracy }=\frac{\mathrm{TP}+\mathrm{TN}}{\mathrm{P}+\mathrm{N}}
$$

In nodules classification process, fusion level classification is also compared with classification by combination of shape, GLCM, FOSF and energy features at different level. Table V shows the comparison of classification by decision fusion and features combination.
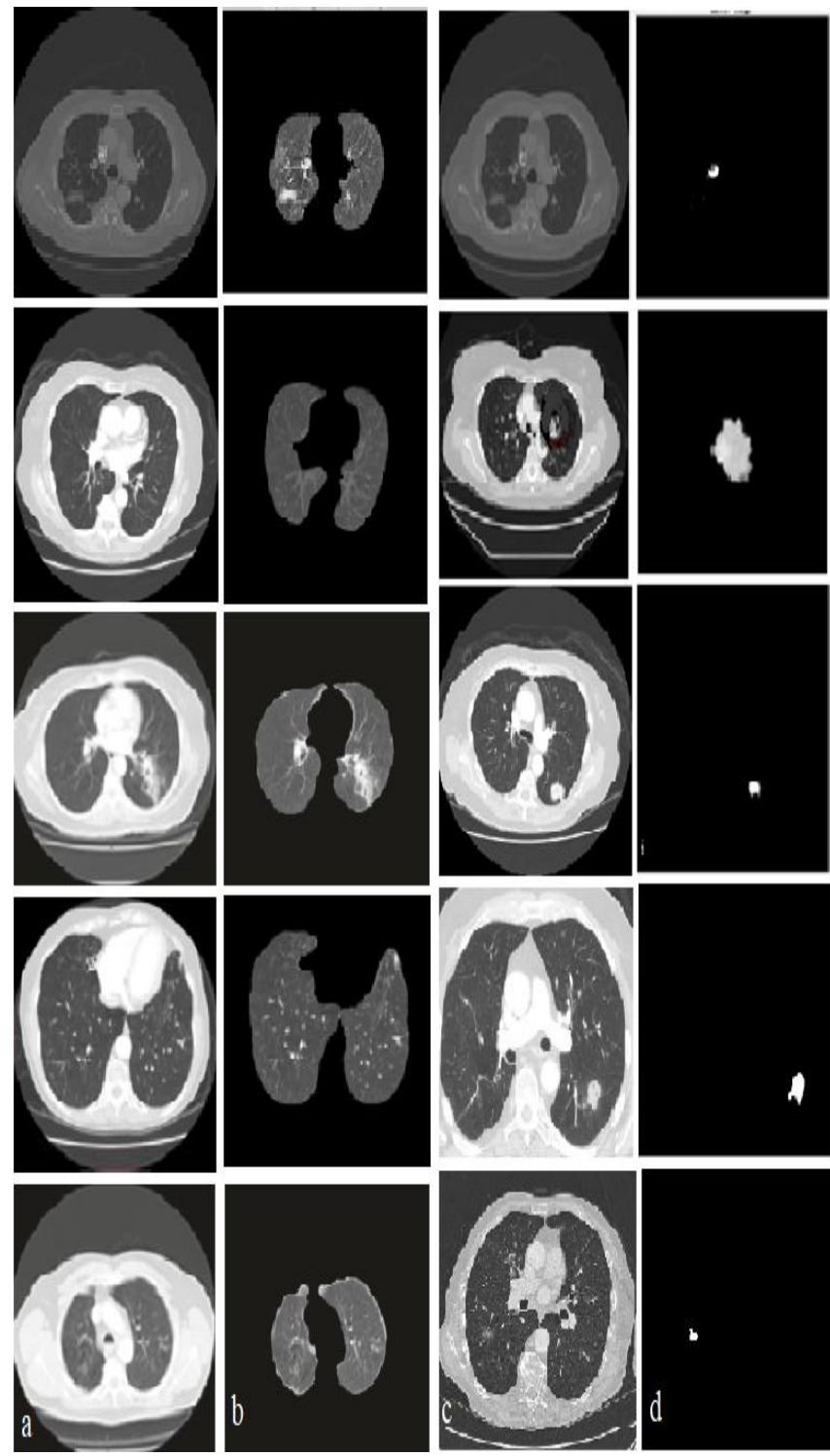

Fig. 4. (a) CT images, (b) segmented lung parenchyma

\begin{tabular}{|c|c|c|c|c|c|}
\hline $\begin{array}{l}\text { Fusin } \\
\text { Level }\end{array}$ & Features & $\begin{array}{l}\text { Acc } \\
(\%)\end{array}$ & $\begin{array}{l}\text { Sen } \\
(\%)\end{array}$ & $\begin{array}{l}\text { Spe } \\
(\%)\end{array}$ & $\begin{array}{l}\text { AUC } \\
(\%)\end{array}$ \\
\hline \multirow{5}{*}{ Features } & $S$ & 88.79 & 87.96 & 89.06 & 87.92 \\
\hline & $\mathrm{F}$ & 86.54 & 86.41 & 86.59 & 85.15 \\
\hline & $\mathrm{G}$ & 89.50 & 89.35 & 89.55 & 90.34 \\
\hline & $\mathrm{E}$ & 85.42 & 84.47 & 84.87 & 88.67 \\
\hline & $\mathrm{D}$ & 90.29 & 91.04 & 90.05 & 92.13 \\
\hline \multirow[b]{4}{*}{ 2-level } & $\mathrm{S}+\mathrm{F}$ & 89.17 & 88.73 & 89.31 & 91.78 \\
\hline & $\mathrm{S}+\mathrm{G}$ & 90.48 & 91.04 & 90.30 & 93.86 \\
\hline & $\mathrm{S}+\mathrm{E}$ & 88.19 & 89.96 & 87.62 & 91.33 \\
\hline & $\mathrm{S}+\mathrm{D}$ & 90.29 & 91.82 & & 0.56 \\
\hline \multicolumn{6}{|c|}{$\begin{array}{l}\text { Published By: } \\
\text { Blue Eyes Intelligence Engineering } \\
\text { \& Sciences Publication }\end{array}$} \\
\hline
\end{tabular}
(c) CT images (c) segmented lung nodules

Table-IV: Performance of decision fusion on dataset 1 
International Journal of Innovative Technology and Exploring Engineering (IJITEE) ISSN: 2278-3075, Volume-9 Issue-2S, December 2019

\begin{tabular}{|c|c|c|c|c|c|}
\hline Fusion & $\mathrm{F}+\mathrm{G}$ & 92.09 & 87.35 & 93.91 & 92.52 \\
\cline { 2 - 6 } & $\mathrm{F}+\mathrm{E}$ & 86.92 & 86.72 & 86.98 & 88.96 \\
\cline { 2 - 6 } & $\mathrm{F}+\mathrm{D}$ & 93.29 & 91.51 & 93.86 & 94.34 \\
\cline { 2 - 6 } & $\mathrm{G}+\mathrm{E}$ & 89.73 & 85.03 & 91.24 & 89.29 \\
\cline { 2 - 6 } & $\mathrm{G}+\mathrm{D}$ & 92.73 & 91.97 & 92.97 & 93.61 \\
\cline { 2 - 6 } & $\mathrm{E}+\mathrm{D}$ & 91.08 & 88.11 & 92.03 & 92.60 \\
\hline \multirow{4}{*}{ 3-level } & $\mathrm{S}+\mathrm{F}+\mathrm{G}$ & 92.35 & 90.74 & 92.87 & 93.04 \\
\cline { 2 - 6 } & $\mathrm{S}+\mathrm{F}+\mathrm{E}$ & 90.55 & 86.57 & 91.83 & 91.94 \\
\cline { 2 - 6 } & $\mathrm{S}+\mathrm{F}+\mathrm{D}$ & 92.24 & 89.66 & 93.07 & 93.67 \\
\cline { 2 - 6 } & $\mathrm{F}+\mathrm{G}+\mathrm{E}$ & 90.18 & 84.86 & 91.98 & 88.98 \\
\cline { 2 - 6 } & $\mathrm{F}+\mathrm{G}+\mathrm{D}$ & 93.25 & 92.43 & 93.51 & 94.17 \\
\cline { 2 - 6 } & $\mathrm{G}+\mathrm{E}+\mathrm{S}$ & 89.95 & 85.80 & 91.29 & 86.35 \\
\cline { 2 - 6 } & $\mathrm{G}+\mathrm{E}+\mathrm{D}$ & 89.73 & 83.79 & 91.63 & 90.02 \\
\cline { 2 - 6 } & $\mathrm{E}+\mathrm{D}+\mathrm{S}$ & 91.56 & 88.73 & 92.47 & 91.98 \\
\cline { 2 - 6 } & $\mathrm{E}+\mathrm{D}+\mathrm{F}$ & 88.94 & 81.94 & 91.92 & 86.88 \\
\cline { 2 - 6 } & $\mathrm{E}+\mathrm{D}+\mathrm{G}$ & 89.90 & 84.72 & 91.43 & 89.56 \\
\cline { 2 - 6 } & $\mathrm{S}+\mathrm{G}+\mathrm{D}$ & 92.16 & 89.50 & 93.20 & 90.63 \\
\hline \multirow{4}{*}{ F-level } & $\mathrm{S}+\mathrm{F}+\mathrm{G}+\mathrm{E}$ & 92.91 & 91.20 & 93.46 & 91.42 \\
\cline { 2 - 6 } & $\mathrm{F}+\mathrm{G}+\mathrm{E}+\mathrm{D}$ & 93.14 & 91.62 & 93.56 & 93.77 \\
\cline { 2 - 6 } & $\mathrm{S}+\mathrm{G}+\mathrm{E}+\mathrm{D}$ & 92.76 & 90.89 & 93.36 & 93.26 \\
\cline { 2 - 6 } & $\mathrm{S}+\mathrm{F}+\mathrm{E}+\mathrm{D}$ & 93.63 & 92.43 & 93.66 & 92.23 \\
\cline { 2 - 6 } & $\mathrm{S}+\mathrm{F}+\mathrm{G}+\mathrm{D}$ & 93.59 & 92.74 & 93.86 & 91.76 \\
\hline 5-level & 5-decision & 95.72 & 95.52 & 95.79 & 96.21 \\
Fusion & $\mathrm{Fusion}$ & & & & \\
\hline
\end{tabular}

\begin{tabular}{|c|c|c|c|c|c|}
\hline & $\mathrm{F}+\mathrm{D}$ & 80.25 & 75.46 & 85.12 & 79.04 \\
\hline & $\mathrm{G}+\mathrm{E}$ & 73.66 & 72.56 & 74.77 & 71.89 \\
\hline & $\mathrm{G}+\mathrm{D}$ & 82.87 & 77.69 & 88.14 & 81.74 \\
\hline & $E+D$ & 77.29 & 74.86 & 79.74 & 72.78 \\
\hline \multirow{11}{*}{$\begin{array}{l}\text { 3-level } \\
\text { Fusion }\end{array}$} & $\begin{array}{l}\mathrm{S}+\mathrm{F} \\
+\mathrm{G}\end{array}$ & 82.46 & 76.87 & 88.14 & 81.94 \\
\hline & $\begin{array}{c}\mathrm{S}+\mathrm{F} \\
+\mathrm{E}\end{array}$ & 71.63 & 69.84 & 73.86 & 72.51 \\
\hline & $\begin{array}{l}\mathrm{S}+\mathrm{F} \\
+\mathrm{D}\end{array}$ & 80.4 & 76.87 & 85.34 & 80.37 \\
\hline & $\begin{array}{c}\mathrm{F}+\mathrm{G} \\
+\mathrm{E}\end{array}$ & 74.78 & 72.71 & 76.88 & 74.74 \\
\hline & $\begin{array}{c}\mathrm{F}+\mathrm{G} \\
+\mathrm{D}\end{array}$ & 86.51 & 83.86 & 89.19 & 84.37 \\
\hline & $\begin{array}{c}\mathrm{G}+\mathrm{E} \\
+\mathrm{S}\end{array}$ & 82.57 & 78.73 & 86.48 & 81.73 \\
\hline & $\begin{array}{l}\text { G+E } \\
+\mathrm{D}\end{array}$ & 81.75 & 75.76 & 87.83 & 84.78 \\
\hline & $\begin{array}{c}E+D \\
+S \\
\end{array}$ & 79.31 & 73.53 & 85.19 & 78.62 \\
\hline & $\begin{array}{c}\mathrm{E}+\mathrm{D} \\
+\mathrm{F}\end{array}$ & 81.97 & 76.72 & 87.31 & 84.38 \\
\hline & $\begin{array}{c}\text { E+D } \\
+G\end{array}$ & 82.8 & 77.84 & 87.83 & 82.45 \\
\hline & $\begin{array}{c}S+G \\
+D\end{array}$ & 87.63 & 85.94 & 89.35 & 84.52 \\
\hline \multirow{5}{*}{$\begin{array}{l}\text { 4-level } \\
\text { Fusion }\end{array}$} & $\begin{array}{c}\mathrm{S}+\mathrm{F} \\
+\mathrm{G}+ \\
\mathrm{E}\end{array}$ & 86.54 & 84.68 & 88.44 & 86.27 \\
\hline & $\begin{array}{c}\mathrm{F}+\mathrm{G} \\
+\mathrm{E}+ \\
\mathrm{D}\end{array}$ & 88.34 & 86.54 & 90.18 & 88.39 \\
\hline & $\begin{array}{c}\mathrm{S}+\mathrm{G} \\
+\mathrm{E}+ \\
\mathrm{D}\end{array}$ & 87.67 & 85.65 & 89.72 & 86.45 \\
\hline & $\begin{array}{c}\mathrm{S}+\mathrm{F} \\
+\mathrm{E}+ \\
\mathrm{D}\end{array}$ & 89.24 & 87.8 & 90.7 & 86.61 \\
\hline & $\begin{array}{c}\mathrm{S}+\mathrm{F} \\
+\mathrm{G}+ \\
\mathrm{D}\end{array}$ & 90.74 & 88.77 & 92.74 & 90.68 \\
\hline $\begin{array}{l}\text { 5-level } \\
\text { Fusion }\end{array}$ & $\begin{array}{c}\text { 5-de } \\
\text { cisio } \\
n \\
\text { Fusi } \\
\text { on }\end{array}$ & 92.54 & 90.48 & 94.63 & 92.69 \\
\hline
\end{tabular}

Table-V: Performance of decision fusion on dataset 2

\begin{tabular}{|l|c|l|l|l|l|}
\hline $\begin{array}{l}\text { Fusin } \\
\text { Level }\end{array}$ & $\begin{array}{l}\text { Feat } \\
\text { ures }\end{array}$ & $\begin{array}{l}\text { Acc } \\
(\boldsymbol{\%})\end{array}$ & $\begin{array}{l}\text { Sen } \\
(\boldsymbol{\%})\end{array}$ & $\begin{array}{l}\text { Spe } \\
(\boldsymbol{\%})\end{array}$ & AUC (\%) \\
\hline \multirow{5}{*}{ Features } & $\mathrm{S}$ & 82.39 & 80.81 & 83.98 & 79.04 \\
\cline { 2 - 6 } & $\mathrm{F}$ & 81.11 & 79.33 & 82.93 & 74.78 \\
\cline { 2 - 6 } & $\mathrm{G}$ & 82.87 & 80.96 & 84.81 & 75.45 \\
\cline { 2 - 6 } & $\mathrm{E}$ & 78 & 75.24 & 80.81 & 80.45 \\
\cline { 2 - 6 } & $\mathrm{D}$ & 83.62 & 76.87 & 90.48 & 83.12 \\
\hline \multirow{5}{*}{$\begin{array}{c}\text { 2-level } \\
\text { Fusion }\end{array}$} & $\mathrm{S}+\mathrm{F}$ & 79.24 & 75.91 & 82.62 & 72.56 \\
\cline { 2 - 6 } & $\mathrm{S}+\mathrm{G}$ & 80.36 & 75.46 & 85.34 & 8127 \\
\cline { 2 - 6 } & $\mathrm{S}+\mathrm{E}$ & 72.34 & 64.44 & 75.3 & 70.67 \\
\cline { 2 - 6 } & $\mathrm{F}+\mathrm{D}$ & 83.58 & 80.74 & 86.48 & 81.66 \\
\cline { 2 - 6 } & $\mathrm{F}+\mathrm{E}$ & 75.98 & 71.07 & 80.99 & 76.53 \\
\cline { 2 - 6 } & & 79.77 & 85.27 & 82.44 \\
\hline
\end{tabular}

Table-VI Performance comparison of decision fusion at different level with features combination on dataset 1

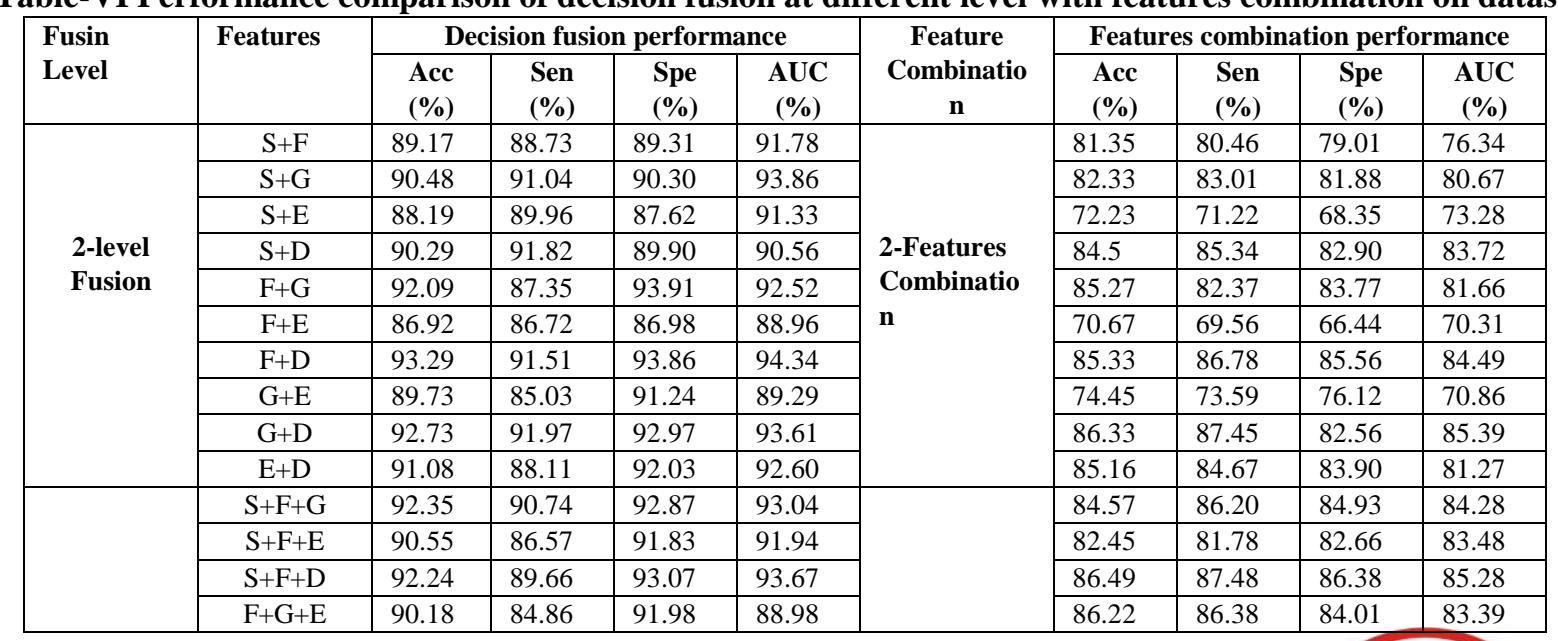




\begin{tabular}{|c|c|c|c|c|c|c|c|c|c|c|}
\hline \multirow{7}{*}{$\begin{array}{l}\text { 3-level } \\
\text { Fusion }\end{array}$} & $\mathrm{F}+\mathrm{G}+\mathrm{D}$ & 93.25 & 92.43 & 93.51 & 94.17 & \multirow{7}{*}{$\begin{array}{l}\text { 2-Features } \\
\text { Combinatio } \\
\text { n }\end{array}$} & 87.39 & 86.93 & 85.49 & 86.17 \\
\hline & $\mathrm{G}+\mathrm{E}+\mathrm{S}$ & 89.95 & 85.80 & 91.29 & 86.35 & & 86.28 & 81.12 & 83.28 & 82.66 \\
\hline & $\mathrm{G}+\mathrm{E}+\mathrm{D}$ & 89.73 & 83.79 & 91.63 & 90.02 & & 84.23 & 83.55 & 84.29 & 83.59 \\
\hline & $\mathrm{E}+\mathrm{D}+\mathrm{S}$ & 91.56 & 88.73 & 92.47 & 91.98 & & 84.78 & 85.28 & 84.31 & 85.34 \\
\hline & $\mathrm{E}+\mathrm{D}+\mathrm{F}$ & 88.94 & 81.94 & 91.92 & 86.88 & & 85.18 & 83.38 & 86.23 & 85.11 \\
\hline & $E+D+G$ & 89.90 & 84.72 & 91.43 & 89.56 & & 86.39 & 85.98 & 78.20 & 79.29 \\
\hline & $\mathrm{S}+\mathrm{G}+\mathrm{D}$ & 92.16 & 89.50 & 93.20 & 90.63 & & 86.32 & 87.20 & 86.93 & 85.29 \\
\hline \multirow{6}{*}{$\begin{array}{l}\text { 4-level } \\
\text { Fusion }\end{array}$} & $\mathrm{S}+\mathrm{F}+\mathrm{G}+\mathrm{E}$ & 92.91 & 91.20 & 93.46 & 91.42 & \multirow{5}{*}{$\begin{array}{l}\text { 2-Features } \\
\text { Combinatio } \\
\text { n }\end{array}$} & 87.28 & 86.86 & 87.31 & 88.19 \\
\hline & $F+G+E+D$ & 93.14 & 91.62 & 93.56 & 93.77 & & 86.29 & 87.37 & 86.96 & 85.77 \\
\hline & $\mathrm{S}+\mathrm{G}+\mathrm{E}+\mathrm{D}$ & 92.76 & 90.89 & 93.36 & 93.26 & & 88.28 & 87.55 & 86.87 & 87.34 \\
\hline & $\mathrm{S}+\mathrm{F}+\mathrm{E}+\mathrm{D}$ & 93.63 & 92.43 & 93.66 & 92.23 & & 89.32 & 85.28 & 83.66 & 86.48 \\
\hline & $\mathrm{S}+\mathrm{F}+\mathrm{G}+\mathrm{D}$ & 93.59 & 92.74 & 93.86 & 91.76 & & 88.41 & 87.55 & 87.38 & 86.85 \\
\hline & $\begin{array}{l}\text { 5-decision } \\
\text { Fusion }\end{array}$ & 95.72 & 95.52 & 95.79 & 96.21 & & 89.59 & 88.38 & 86.31 & 85.84 \\
\hline
\end{tabular}

\section{CONCLUSION}

In this research study, fully automated CADe/CADx system was proposed for the segmentation and classification of WCNs, JVNs and JPNs on CT images. By means of proposed system lung nodules detection along with classification was performed with high accuracy, sensitivity, specificity and AUC. Moreover, our previously designed algorithms for JVNs and JPNs segmentation were used. In classification process five set of features texture, shape, first order statistics, energy and deepCNN were extracted from segmented nodules. Five individually trained PNNs with separated set of features were used as classifier for nodules classification. PNNs classification decisions were fused at different levels such as, 2-level, 3-level, 4-level and 5-level to achieve the accurate classification. Evaluation results showed that classification accuracy is highest at 5-level fusion. Proposed system was evaluated on CT images from LIDC database. Total of 2669 nodules were considered with malignancy rate of 1 to 5 . On data set 1 with nodules of malignancy rate 1, 2 and 3 our proposed 5-level fusion achieved the accuracy of 95.72, sensitivity of 95.52, specificity of 95.79 and Area Under Curve of 96.2. For dataset 2 with nodules of malignancy rate 3,4 , and 5 it achieved accuracy of 92.54 , sensitivity of 90.48 , specificity of 94.63 and Area Under Curve of 92.69 for dataset 2 . Performance on dataset 2 was less as compared against dataset2.

\section{REFERENCES}

1. Amal, A.F.; Ali, A.; Elshazly, S.; Farag, A.A. Feature fusion for lung nodule classification. Int. J. Comput. Assist. Radiol. Surg. Vol. 12, 2017, 1809-1818.

2. Dhara A. K, Mukhopadhyay S, Dutta A, Garg M and Khandelwal N, " A Combination of Shape and Texture Features for Classification of Pulmonary Nodules in Lung CT Images”. J. Digit. Imaging, vol. 29, 466-475.

3. J. Kuruvilla and K. Gunavathi, "Lung cancer classification using neural networks for CTimages," Computer Methods and Programs in Biomedicine, vol. 113, no. 1, pp. 202-9, 2014.

4. M. Z. ur Rehman, M. Javaid, S. I. A. Shah, S. O. Gilani, M. Jamil, and S. I. Butt, "An appraisal of nodules detection techniques for lung cancer in CT images" Biomedical Signal Processing and Control, vol. 41, pp. 140-151, 2018.

5. Xie Y.T., Zhang J.P., Xia Y. Zhang and Y.N. "Fusing texture, shape and deep model-learned information at decision level for automated classification of lung nodules on chest CT". Inf. Fusion, vol. 42, 102-110, 2018

6. Wei G.H, Cao H, Ma H, Qi S.L, Qian W and Ma Z.Q "Content-based image retrieval for Lung Nodule Classification Using Texture Features and Learned Distance Metric”. Journal. Med. Syst. 2018, vol. 42, no. 13 2018 .

7. Zhu W.T, Liu C.C, Fan W and Xie X. H, "DeepLung: 3D Deep Convolutional Nets for Automated Pulmonary Nodule Detection and Classification". arXiv, 2017.

8. Kim B. C, Sung Y. S and Suk H. L, "Deep feature learning for pulmonary nodule classification in a lung CT". In Proceedings of the 2016 4th International Winter Conference on Brain-Computer Interface (BCI), Yongpyong, South Korea, 1-3, 2016.

9. Lin P.L, Huang P.W, Lee C.H, and Wu M.T, “Automatic classification for solitary odpulmonary nule in CT image by fractal analysis based on fractional Brownian motion model. Pattern Recognit. vol.46, 3279-3287, 2013

10. Nascimento L. B, de Paiva A and Silva A.C, "Lung Nodules Classification in CT Images Using Shannon and Simpson Diversity Indices and SVM". In Proceedings of the MLDM, Machine Learning and Data Mining, Pattern Recognition, Berlin, Germany, 454-466. 2012

11. Chen H, Wu W.F, Xia H, Du J, Yang M and Ma, "Classification of Pulmonary Nodules Using Neural Network Ensemble". In Proceedings of the 8th International Conference on Advances in Neural Networks, Guilin, China, vol. 3, 460-466, 2011.

12. Dilger S.K, Judisch A, Uthoff J, Hammond E, Newell J. D and Sieren J.C. "Improved pulmonary nodule classification utilizing lung parenchyma texture features". In Proceedings of the Medical Imaging: Computer-Aided Diagnosis, 21-26,Orlando, FL, USA, 2015.

13. Way T.W, Sahiner B and Chan H. P, "Computer-aided diagnosis of pulmonary nodules on CT scans: Improvement of classification performance with nodule surface features". Med. Phys. Vol. 36, , 3086-3098. 2009.

14. Jiang H.Y, Ma H, Qian W, Wei G.H, Zhao X.Z and Gao M. "A novel pixel value space statistics map of the pulmonary nodule for classification in computerized tomography images". In Proceedings of the 2017 39th Annual International Conference of the IEEE Engineering in Medicine and Biology Society (EMBC), Jeju Island, Korea, 556-559, 2017.

15. Rendon-Gonzalez E and Ponomaryov V. "Automatic Lung nodule segmentation and classification in CT images based on SVM". In Proceedings of the 2016 9th International Kharkiv Symposium on Physics and Engineering of Microwaves, Millimeter and Submillimeter Waves (MSMW), Kharkiv, Ukraine, 2016.

16. Way T.W, Hadjiiski L. M and Sahiner B. "Computer-aided diagnosis of pulmonary nodules on CT scans: Segmentation and classification using 3D active contours". Med. Phys. vol. 33, 2323-2337, 2006.

17. Armato S. G, Altman M. B and Wilkie J. "Automated lung nodule classification following automated nodule detection on CT: A serial approach". Med. Phys. vol. 3, 1188-1197, 2003.

18. Dandil E, Çakiroglu M, Eksi , Ozkan M, Kurt O.K and Canan A. "Artificial neural network-based classification system for lung nodules on computed tomography scans". In Proceedings of the $20146^{\text {th }}$ International Conference of Soft Computing and Pattern Recognition (SoCPaR), Tunis, Tunisia, 382-386, 2014.

19. Htwe K.Z, Yamamori K, Katayama T and Kyi T.M. "Automated lung nodule classification by artificial neural network and fuzzy inference system". In Proceedings of the 2016 IEEE 5th Global Conference on Consumer Electronics (GCCE), Kyoto, Japan, 2016. 
20. Shen W, Zhou M, Yang F, Yu D. D, Dong, Di, Yang C.Y, Zang Y.L and Tian J. "Multi-crop Convolutional Neural Networks for lung nodule malignancy suspiciousness classification". Pattern Recognit. vol. 61, 663-673, 2017.

21. Zhao X. Z, Liu L.Y, Qi S.L, Teng Y.Y, Li J.H and Qian, W. "Agile convolutional neural network for pulmonary nodule classification using CT images”. Comput. Assist. Radiol. Surg. Vol. 18, 585-595, 2018.

22. Jiang H.Y, Ma H, Qian W, Gao M.D and Li Y. "An Automatic Detection System of Lung Nodule Based on Multigroup Patch-Based Deep Learning Network". IEEE J. Biomed. Health Inform. vol. 22, 1227-1237, 2018

23. S.G.A. Iii, G. Mclennan, L. Bidaut, M.F. Mcnittgray, C.R. Meyer, A.P. Reeves, et al., The lung image database consortium (LIDC) and image database resource initiative (IDRI): A completed reference database of lung nodules on CT scans, Medical Physics, 38 (2) (2011) 915-931.

24. LIDC-IDRI. The Cancer Imaging Archive.

25. Mekali V., Girijamma H.A. (2019) Automated Lung Nodules and Ground Glass Opacity Nodules Detection and Classification from Computed Tomography Images. In: Pandian D., Fernando X., Baig Z., Shi F. (eds) Proceedings of the International Conference on ISMAC in Computational Vision and Bio-Engineering 2018 (ISMAC-CVB). ISMAC 2018. Lecture Notes in Computational Vision and Biomechanics, vol 30. Springer, Cham.

26. Vijayalaxmi Mekali, H. A. Girijamma, "An Fully Automated CAD System for Juxta-Vacular Nodules Segmentation in CT Scan Images", IEEE Trans. in 3rd International Conference on Computing Methodologies and Communication (ICCMC), 2019.

27. Vijayalaxmi Mekali, Girijamma H. A, "Fully Automatic Detection and Segmentation Approach for Juxta-Pleural Nodules from CT Images", IJHISI.

\section{AUTHORS PROFILE}

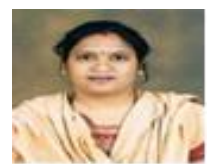

Mrs. Vijayalaxmi Mekali received masters degree (M.Tech) from VTU, Belagavi. She is Assistant Professor at Department of CSE, K. S. I. T, Karnataka, India. Currently, she is pursuing. Ph. D in VTU, under guidance of Dr. Girijamma H A, Prof. R.N.S Institute of Technology, Bangalore. Her current research topic includes medical image processing.

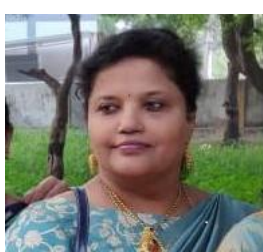

Dr. Girijamma H A received $\mathrm{Ph}$. D from Kuvempu University in 2012 She is Professor at Department of Computer Science and Engineering, R.N.S Institute of Technology, Bangalore. Her current research topics includes fuzzy logic, medical image processing and machine learnin 